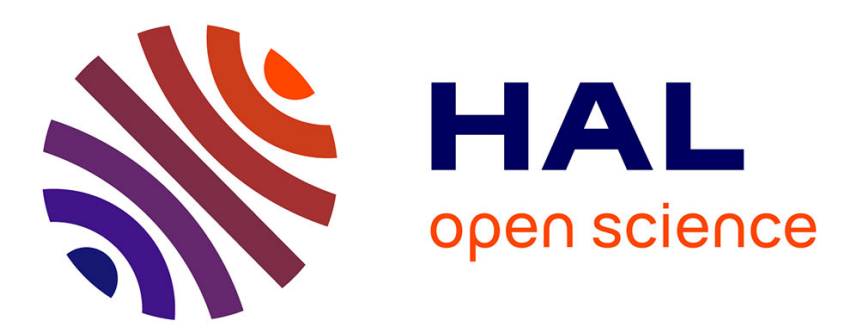

\title{
Some Challenges and Opportunities in Reliability Engineering
}

Enrico Zio

\section{To cite this version:}

Enrico Zio. Some Challenges and Opportunities in Reliability Engineering. IEEE Transactions on Reliability, 2016, 65 (4), pp.1769-1782. 10.1109/TR.2016.2591504 . hal-01550063

\section{HAL Id: hal-01550063 \\ https://hal.science/hal-01550063}

Submitted on 29 Jun 2017

HAL is a multi-disciplinary open access archive for the deposit and dissemination of scientific research documents, whether they are published or not. The documents may come from teaching and research institutions in France or abroad, or from public or private research centers.
L'archive ouverte pluridisciplinaire HAL, est destinée au dépôt et à la diffusion de documents scientifiques de niveau recherche, publiés ou non, émanant des établissements d'enseignement et de recherche français ou étrangers, des laboratoires publics ou privés. 


\title{
Some challenges and opportunities in reliability engineering
}

\author{
Enrico Zio ${ }^{\mathrm{a}, \mathrm{b}}$ \\ a'Chair System Science and the Energy Challenge, Fondation Electricite' de France (EDF), CentraleSupélec, Université \\ Paris-Saclay, Grande Voie des Vignes, 92290 Chatenay-Malabry, France \\ ${ }^{\mathrm{b}}$ Dipartimento di Energia - Politecnico di Milano \\ Via La Masa 34, I-20156 Milano, Italy
}

\begin{abstract}
Today's fast-pace evolving and digitalizing World is posing new challenges to reliability engineering. On the other hand, the continuous advancement of technical knowledge and the increasing capabilities of monitoring and computing offer opportunities for new developments in reliability engineering.

In this paper, I reflect on some of these challenges and opportunities in research and application. The underlying perspective taken stands on:

- the belief that the knowledge, information and data (KID) available for the modeling, computations and analyses done in reliability engineering is substantially grown and continue to do so;

- the belief that the technical capabilities for reliability engineering have been significantly advanced;

- the recognition of the increased complexity of the systems, nowadays more and more made of heterogeneous, highly interconnected elements.

In line with this perspective, opportunities and challenges for reliability engineering are discussed in relation to degradation modeling and integration of multi-state and physics-based models therein, accelerated degradation testing, component-, system- and fleet-wide prognostics and health management in evolving environments.

The paper is not a review, nor a state of the art work, but rather it offers a vision of reflection on reliability engineering, for consideration and discussion by the interested scientific community. It does not pretend to give the unique view, nor to be complete in the subject discussed and the related literature referenced to.
\end{abstract}


Keywords: Degradation Modeling, Physics-Based Models, Dependent Degradation, Random Shocks, Multi-State System Reliability, Piecewise Deterministic Markov Process, Accelerated Degradation Testing, Prognostics and Health Management, Prognostic Performance Indicator, Return of Investment, Evolving Environment, Fleet Prognostics, Distributed Prognostics

\section{List of acronyms}

AAKR Auto-Associative Kernel Regression

ADT Accelerated Degradation Testing

AIC Akaike's Information Criterion

ALT Accelerated Life Testing

ANN Artificial Neural Network

BBD Binary Decision Diagrams

BMA Bayesian Model Averaging

CBM Condition-Based Maintenance

CL Cost of Loss

CM Corrective Maintenance

CMS Condition Monitoring System

D-S theory Dempster-Shafer theory

EE Evolving Environment

FA Failures Avoided

HI Health Index

KID Knowledge, Information and Data

KNN K-Nearest Neighbors

LED Light Emitting Diode

MOSFET Metal Oxide Semiconductor Field Effect Transistor

MSM Multi-State Model 
MSPM Multi-State Physics Model

PBM Physics-Based Model

PDMP Piecewise Deterministic Markov Processes

PHM Prognostics and Health Management

PI Precision Index

PrM Preventive Maintenance

PMS Phased-Mission Systems

PPI Prognostic Performance Indicator

RI Risk Index

ROI Return On Investment

RUL Remaining Useful Life

RVM Relevance Vector Machines

SPRT Sequential Probability Ratio Test

SVM Support Vector Machines 


\section{Introduction}

As the digital, physical and human worlds continue to integrate, we experience a deep transformation in industry, which far-reaches into our lives. The $4^{\text {th }}$ industrial revolution, the internet of things and big data, the industrial internet, are changing the way we design, manufacture, provide products and services. This is creating a complex network of things and people that are seamlessly connected and communicating. It is providing opportunities to make productions systems more efficient and faster, and more flexible and resilient the complex supply chains and distribution networks that tie the global economy.

In this fast-pace changing environment, the attributes related to the reliability of components and systems continue to play a fundamental role for industry. The innovations that are being developed have high potential of increased wellbeing and benefits, but also generate new and unknown failure mechanisms, new and unknown functional and structural dependencies, and eventually new and unknown hazards and risks. On the other hand, the advancements in knowledge, methods and techniques, the increase in information sharing and data availability, offer new opportunities of analysis and assessment for reliability engineering. Then, a new reliability engineering "revolution" is in the making for addressing the challenges brought about by the new and evolved systems, and the innovations therein; this calls for and, at the same time, drives the advancements of new methods and tools, and the extension of their applications, based on the increased knowledge, information and data (KID) available, which can improve our reliability prediction capability.

In this paper, I consider the above context and address some challenges and opportunities for reliability engineering. In particular, I argue that the big KID available allows refined modeling of the degradation processes that drive the components and systems to failure.

In Section 2, multi-state modeling schemes are discussed as an opportunity of describing the degradation processes as Piecewise Deterministic Markov Processes (PDMP), accommodating the relevant knowledge in physics-based models of transition rates (Multi-State Physics-based Models, MSPM). Challenges are highlighted in relation to the treatment of dependent, and often competing, processes, the accounting of uncertainties and of the effects of maintenance. On the contrary, the complexity of the resulting models does not impede their solution by advanced mathematical techniques and Monte Carlo simulation, although scalability may be a challenge for large systems. As for the problem of the estimation of the model parameters values, this can be tackled by Accelerated Degradation Testing (ADT), as discussed in the subsequent Section 3.

On the other hand, the increased availability of data coming from monitoring the relevant components and systems parameters and the grown ability of treating these data by intelligent 
algorithms capable of mining out information relevant to the assessment and prediction of their state, has open wide the doors for Prognostics and Health Management (PHM) in many industrial sectors, for improved operation and maintenance. This is discussed in Section 4, highlighting the challenges of:

- developing methods and models capable of dealing with the inevitable Evolving Environment (EE) that changes the conditions in which a component or system lives, and degradation and failure occur (PHM in EE);

- considering the information and data coming from a fleet of components and systems, for effectively informed PHM (Fleet PHM);

- considering the integration and interaction of the information and data monitored on multiple components operating on a system (Distributed PHM);

- evaluating the performance of PHM approaches by defining significant Prognostic Performance Indicators (PPIs) and their adequacy for a given application, by a maturity assessment of the predictive model;

- evaluating the economic and safety value of a PHM approach for condition-based and/or predictive maintenance.

\section{Degradation modeling}

In the evolving scenario of development of complex networks of things and people, supported by (more and more interdependent) critical infrastructures, concerns are arising on vulnerability to failure and risk of accident. The worries are that the allocated system capacities may not be adequate to respond to the postulated growing demands and that the safety margins preventively designed may degrade and become insufficient to cope with the expected and unexpected stresses arriving onto the systems. Emergent behaviors may arise in collective ways difficult to predict from the superposition of the behavior of the individual elements and difficult to manage resiliently. Indeed, we are witnessing more and more system-level breakdowns in our critical infrastructures, which emerge from local degradations of individual components that cascade to large-scale consequences possibly propagating from one system to another, through the (inter-)dependencies. The analysis of this problem for ensuring the protection and resilience of our critical infrastructures calls for the integration of methods capable of viewing the problem from different perspectives (topological and functional, static and dynamic, etc.), under the existing uncertainties and given the high system complexity (Kröger and Zio 2011).

On the other hand, safety-critical components and systems, like those employed in the nuclear, oil 
and gas, automotive, aeronautic and aerospace sectors, are designed not to fail, i.e. with very high reliability, because of the potentially catastrophic consequences of their failures. Traditional databased reliability analysis, based on failure data, is, then, unsuitable. Yet, most failure mechanisms can be traced to underlying degradation processes (e.g. wear, stress corrosion, shocks, cracking, fatigue, etc.), for which models may exist (Yang, 2002).

In general, the reliability of a system decreases as the degradation processes develop, eventually leading to failure (Ye and Xie, 2015). In reliability engineering, degradation processes have been widely studied and different degradation models have been developed.

However, there is a need for the development of a holistic framework of models and computational methods for the reliability-based analysis and maintenance optimization of safety-critical systems, taking into account the available knowledge about the components and systems degradation and failure behaviors, their dependencies, the external influencing factors and the associated uncertainties. The holistic treatment of the problem should also allow for the consideration of damage precursor, as backbones for tracking component and system degradation which can allow early detection and effective, predictive maintenance.

The existing degradation models are often classified into the following general categories:

- statistical models of time to failure, based on degradation data (e.g. Bernstein distribution (Gebrraeel et al., 2009), Weibull distribution (Lu and Meeker, 1993));

- stochastic process models (e.g. Gamma process (Lawless and Crowder, 2004), inverse Gaussian process (Chen et al., 2015)) describing the evolution of one or more degradation parameters by gradual, stochastic degradation increments over time, and the failure occurs when the degradation parameter values reach predefined thresholds;

- physics-based models (PBMs), based on the knowledge of the physics of degradation, which is translated into equations to give a quantitative description (e.g. the physics functions based on critical environmental stresses, e.g. amplitude and frequency of mechanical loads, used to model the pitting and corrosion-fatigue degradation mechanisms (Chookah et al, 2011));

- multi-state models (MSMs) describing the underlying degradation process by finite degradation states (e.g. semi-Markov models for the deterioration of infrastructure systems (Black et al., 2005); piecewise deterministic Markov process for dependent degradation process (Lin et al., 2014)).

The recent literature on degradation modeling can be organized under the above taxonomy. For statistical models, Lu et al. (Lu et al., 1997) have combined random regression coefficients and a standard deviation function for analyzing linear degradation data for statistical inference of a time-to- 
failure distribution. $\mathrm{Lu}$ and Meeker (Lu and Meeker, 1993) have developed methods using degradation measures to estimate a time-to-failure distribution for a broad class of degradation models and demonstrated some special cases for which it is possible to obtain closed-form expressions of the distributions. Yang and Yang (Yang and Yang, 1998) have estimated the parameters of lifetime distributions using a random-coefficient-based approach that uses the lifetimes of failed devices, combined with degradation information from operating devices.

For stochastic models, Whitmore (Whitemore, 1995) has estimated the degradation process by a Wiener diffusion process subject to measurement errors due to imperfect instruments, procedures and environments. Lawless and Crowder (Lawless and Crowder, 2004) have constructed a tractable Gamma-process model incorporating a random effect for taking into account different degradation rates of the individual components. Chen et al. (Chen et al., 2015) have employed the inverse Gaussian process with random-drift mode, in which the random drifts are used to represent heterogeneities commonly observed across the product population. Note that the aforementioned degradation models are always built on sufficient degradation/failure data.

Physics-Based Models (PBMs) (Daigle and Goebel, 2011; Reggiani et al., 2011; Keedy and Feng, 2012) and Multi-State Models (MSMs) (Moghaddass and Zuo, 2014; Lisnianski and Levitin, 2003; Li and Pham, 2005; Lin et al., 2012) can be used to describe the evolution of degradation in structures, systems and components, for which statistical degradation/failure data are insufficient, e.g. the highly reliable devices in the nuclear, oil and gas, automotive, aeronautic and aerospace industries. For PBMs, Daigle and Goebel (Daigle and Goebel, 2011) have developed a physics model of a pneumatic valve, based on mass and energy balances in which the damages depend on sliding velocity. Reggiani et al. (Reggiani et al., 2011) have developed a physics-based analytical expression of the linear drain current for hot-carrier stress degradation in transistors. Keedy and Feng (Keedy and Feng, 2012) have proposed a probabilistic reliability and maintenance modeling framework for stent deployment and operation, based on physics-of-failure mechanisms, e.g. delayed failure due to fatigue crack and instantaneous failure due to overload fracture.

For MSMs, Moghaddass and Zuo (Moghaddass and Zuo, 2014) have employed the nonhomogeneous continuous-time hidden semi-Markov process to model the degradation and observation processes associated with the device. Giorgio et al. (Giorgio et al., 2011) have developed an age- and statedependent Markov model for the wear process of cylinder liners of identical heavy-duty diesel engines for marine propulsion. Unwin et al. (Unwin et al., 2011) have proposed a multi-state physics model (MSPM) for the cracking process of a dissimilar metal weld in a primary coolant system of a nuclear power plant.

Yet, there are several factors which can influence degradation evolution and, thus, need to be accounted for in degradation modeling. 
Indeed, in practice components and systems are often subject to multiple competing degradation processes and any of them may cause failure (Wang and Pham, 2012). The dependencies among these processes within one component (e.g. the wear of rubbing surfaces influenced by the environmental stress shock within a micro-engine (Jiang et al., 2012)), or/and among different components (e.g. the degradation of the pre-filtrations stations leading to a lower performance level of the sand filter in a water treatment plant (Rasmekomen and Parlikad, 2013)) need to be considered, under certain circumstances. Components can be dependent due to functional dependence, where the failure of a trigger component causes other components to become inaccessible or unusable (Xing et al., 2012; Wang et al., 2012). Failure isolation effects can induce degradation dependency among different components, since failure of one component may cause other components within the same system to become isolated from the system due to the failure isolation actions (Xing and Levitin, 2010; Wang et al., 2013). This renders challenging the analysis and prediction of the components and systems reliability (Peng et al., 2010). Wang and Pham (Wang and Pham, 2012) applied time-varying copulas for describing the dependencies between the degradation processes modeled by statistical distributions. Straub (Straub, 2009) used a dynamic Bayesian network to represent the dependencies between degradation processes modeled by multistate models. However, very few studies have considered degradation dependency in a system whose degradation processes are modeled by PBMs and MSMs.

Components may also suddenly fail due to randomly occurring events of excessive loading or environmental conditions (e.g. excessive temperature) (Wang et al., 2011). For example, thermal and mechanical shocks (e.g. internal thermal shocks and water hammers) (Lydell, 2000; Salonen et al., 2007) onto power plant components can lead to intense increases in temperatures and stresses, respectively. These events, referred to as random shocks, need to be accounted for on top of the underlying degradation processes, because they can contribute to accelerating the degradation processes. In the literature, random shocks are typically modeled by Poisson processes (Li and Pham, 2005), distinguishing two main types, extreme shock and cumulative shock processes (Bai et al., 2006), according to the severity of the damage. The former could directly lead the component to immediate failure (Anderson, 1987), whereas the latter increases the degree of damage in a cumulative way (Agrafiotis and Tsoukalas, 1995). Esary and Marshall (1973) have considered extreme shocks in a component reliability model, whereas Wang et al. (2011), Klutke and Yang (2002) and Wortman et al. (1994) have modeled the influences of cumulative shocks on a degradation process. Both extreme and cumulative random shocks have been considered by Li and Pham (2005), and Wang and Pham (2012). Additionally, Ye et al. (2011) and Fan et al. (2000) have considered that a high severity of degradation can lead to a high probability that a random shock causes extreme damage. However, the fact that the effects of cumulative shocks can vary according to the severity of degradation has also to be considered. 
Besides, previous research has focused on the dependency between continuous/multi-state degradation processes and random shocks. For continuous degradation processes, Peng et al. (2010) considered systems with one linear degradation path where shocks can bring additional abrupt degradation damage if the shock loads do not exceed the maximum strength of the material. Multicomponent systems subject to multiple linear degradation paths have been further considered by Song et al. (2014). Jiang et al. (2012) studied changes in the maximal strength of the material when systems are deteriorating under different situations. Becker et al. (2002) extended the theory of dynamic reliability to incorporate random changes of the degradation variables due to random shocks. Rafiee et al. (2014) proposed reliability models for systems for which the degradation path has a changing degradation rate according to particular random shock patterns. Song et al. (2014) studied random shocks with specific sizes or functions, which can selectively affect the degradation processes of one or more components (not necessarily all components) in one system. For multi-state degradation processes, Yang et al. (2011) combined random shocks with Markov degradation models where shocks can lead the systems to further degraded states. However, few studies have explicitly considered both the dependencies between degradation processes and the random shocks, and among the degradation processes themselves.

Finally, it is important to consider that the degradation processes can be interrupted or slowed down by maintenance tasks (e.g. one component can be restored to its initial state by preventive maintenance if any of its degradations exceed the respective critical level (1992) and by corrective maintenance upon its failure (2012)). The interactions among components complicate the modeling for maintenance planning, which becomes a big challenge (2008). Thomas (1986) has categorized these interactions in maintenance modeling into three groups: economic, structural and stochastic dependences. Economic dependence exists when the maintenance cost of several components is not equal to the sum of their individual maintenance costs. For example, Castanier et al. (2005) have considered a condition-based maintenance policy for a two-unit deteriorating system, where the setup cost of inspection is charged only once if the actions on the two components are combined. Van Dijkhuizen (2000) has investigated the long-term grouping of preventive maintenance jobs in a multi-setup, multi-component production system where the set-up activities can be combined when several components are maintained at the same time. Structural dependence occurs if some working components need to be replaced or dismantled in order to execute the maintenance of the failed ones. For example, Dekker et al. (1998) have studied the maintenance policy for asphalt roads, where the number of maintenance services is limited by integrating neighboring segments into a homogeneous section which is completely repaired. Stochastic dependence, also referred to as probabilistic dependence, applies when the state of one component can affect those of other components or their failure rates. Failure interactions have been the most discussed cases for stochastic dependence (Rasmekomen and Parlikad, 2013) and imply that the failure of one component may lead to the failure of other components with certain probabilities, and/or influence their failure rates (Murthy 
and Nguyen, 1985). For example, Lai and Chen (Lai and Chen, 2006) have presented an economic periodic replacement model for a two-unit system where the failure of unit 1 can increase the failure rate of unit 2, while the failure of unit 2 induces unit 1 into instantaneous failure. Zequeira and Bérenguer (2005)] have studied the inspection policies for a two-component standby system, where the failure of one component can modify the conditional failure probability of the component still in operation with probability $p$ and does not modify it with probability $1-p$. Barros et al. (2006) have optimized the maintenance policy for a two-unit parallel system where the failure of a component increases the failure rate of the surviving one.

Dependency among degradation mechanisms or processes has received less attention within the framework of maintenance modeling and optimization of multi-component systems, although they are of real concern in practice (e.g. the failure of a pump due to oxidation of contacts and bear wearing). Peng et al. (2010) have developed a maintenance policy with periodic inspections when two dependent or correlated failure processes are considered. Jiang et al. (2012) have further compared two preventive maintenance (PrM) policies, age replacement policy and block replacement policy, combining immediate corrective replacement in consideration of shifting failure thresholds. Özekici (1988) has considered interdependent aging processes between components due to continuous wear and shocks, and proposed an optimal periodic replacement policy. Rasmekomen and Parlikad (2013) have considered degradation dependency in terms of output performance between one critical component and other parallel components based on aging processes, and the optimal agebased maintenance policy for this case was also studied. Yang et al. (2013) have proposed a general statistical reliability model for repairable multi-component systems considering dependent competing risks, under a partially perfect repair assumption which considers that only the failed component, rather than the whole system, is replaced. Hong et al. (2014) have used copulas to model degradation dependency among all the components of a system and obtained the optimal maintenance policy including condition-based maintenance with periodic inspections and instantaneous corrective maintenance (CM). Van Horenbeek and Pintelon (2013) have proposed a dynamic predictive maintenance policy that minimizes the long-term mean maintenance cost per unit time while considering different component dependencies (i.e. economic, structural and stochastic dependence). Song et al. (2014) have applied age replacement policy and inspection-based maintenance policy for systems whose components have $s$-dependent failure times, and the optimal replacement interval or inspection times are determined. Note that maintenance optimization for multi-component systems with multiple dependent competing degradation processes within individual components has not been considered and only the pre-scheduled periods for inspection or maintenance are considered as the decision variables of the optimization problem.

From the above description and discussion of the degradation processes, and the associated relevant 
factors of dependency, random shocks and maintenance effects, it seems evident that a componentlevel and system-level, holistic framework of models and computational methods of reliability analysis and maintenance optimization is necessary to integrate the available data and knowledge on degradation processes and failure mechanisms, their dependencies, the external influencing factors and the associated uncertainties. One main argument in support to this relates to the need of considering dependencies among different aspects of the components and systems degradation processes (dependencies among multiple competing degradation processes within one component or among different components, functional dependencies among components, etc.). It would, indeed, be very advantageous to have a holistic degradation modelling framework that allows considering in an integrated manner all such dependencies at component-level and system-level. More specifically, the availability of such modeling framework would be strongly beneficial for the asset management of these components and systems, because it would enable to realistically predict component and system degradation, and optimally plan the necessary maintenance activities. However, it is important to recognize that at the current state of practice, it is usually not feasible to integrate the consideration of all these aspects and incorporate them in a single application problem. For practicality purposes, modeling solutions must be developed, that enable such holistic description of the degradation processes occurring in a component or system, and their quantitative evaluation by efficient computation.

Finally, such modelling framework would be apt to deal with phased-mission systems (PMS), such as those encountered in nuclear, aerospace, chemical, electronic and other industries. These systems are required to perform different tasks either during any given mission or their operational life time. For example, a modern aircraft flight typically involves automated take-off, ascent, level flight, altered flight due to interferences, descent and landing where each of these phases may require different configurations of components and develop under different environments. In a boiling water reactor, a loss of coolant accident involves three phases for emergency core cooling: initial core cooling, suppression core cooling, and residual heat removal. Other systems, such as communication satellites, may require transportation to the operation site, followed by deployment and a multitude of on-station activities. Different systems or system configurations may be used during different phases in different environments, under varying degrees of stress and with consequent different degradation evolution. In some cases, under certain conditions, a degraded system may be capable of continuing the phases until mission completion. The reliability analysis of phased-mission systems must, then, account for changes in configuration, component use, stresses and degradation evolution. Models of PMS can be built using combinatorial models, Fault Trees, Markov models, Petri nets, Binary Decision Diagrams (BDDs), simulation models and others (Alam and Al-Saggaf, 1986; Bondavalli et al., 2004; Dong et al., 2008; Dugan, 1991; Fussel et al., 1981; Lai and Chen, 2006; Laskey, 1996; Mura and Bondavalli, 1999 and 2001; Sormani et al., 1992; Smotherman and Zermoudeh, 1989; Wang et al., 2012; Xing and Dugan, 2002 and 2004; Xing and Levitin, 2013; Yong and Dugan, 2004; 
Zang et al., 1999). During a particular phase, only certain system features are important, in accordance with the specific task(s) being performed in that phase. Typically, each phase is identified by: phase number; duration; system configuration or task(s) to be performed; performance measure(s) of interest; and maintenance policy. The whole mission is described by a mission profile table. The overall reliability of a PMS is the probability that the mission successfully achieves (all) the submission objectives in each phase.

In the context of PMS reliability analysis, the modeling framework discussed above would be quite useful to capture the relevant factors of dependency, random shocks and maintenance effects, pertaining to each different phase over the entire mission duration, within an integrated analysis for the calculation of system and mission performance indicators such as the probability of mission success, the mean time to failure, the ranks of importance of the system features in the different phases of the mission.

\section{Accelerated Degradation Testing}

As mentioned in the previous Section, many components and systems, particularly those employed in safety-critical applications, are designed to be highly reliable and to have a long lifespan, e.g. battery life of 15 years for hybrid electric vehicles (Chalk and Miller, 2006).

Traditional reliability tests are obviously not suitable for the reliability assessment of such equipment over such long time spans. Thus, accelerated degradation tests (ADT) are widely used to accelerate the failure/degradation processes, exposing the equipment to severe test conditions. Successful applications of ADT have been developed for batteries (Thomas et al., 2008), light emitting diodes (LED)s (Wang and Chu, 2012), metal oxide semiconductor field effect transistors (MOSFETs) (Santini et al., 2014), and others.

In standard ADT data analysis, a degradation model like those discussed in the previous Section is assumed to describe the degradation paths of the samples tested at different stress levels and some specific parameters of the model are assumed to be stress-related, as described by a given acceleration model, e.g. the drift coefficient in the Wiener process (Whitemore and Schenkelberg, 1997; Park and Padgett, 2006; Lim and Yum, 2011). In general, acceleration models are assumed based on the physical mechanisms of the tested samples or empirical observations of the stress variable (Escobar and Meeker, 2006), e.g. temperature-Arrhenius model, voltage-Eyring model, etc. After obtaining the data from ADT, statistical inferences are made to get estimates of the unknown parameters in both degradation and acceleration models. Then, the component reliability assessment and lifetime evaluation are performed with the estimated parameters, and considering the given use 
conditions. Statistical inference methods for ADT data analysis have been extensively reviewed in Nelson (1990) and Meeker and Escobar (1998).

In literature, stochastic process models have drawn more attention than degradation-path models, because of their properties of time-dependent structures, like the Wiener (Whitemore and Schenkelberg, 1997; Lim and Yum, 2011; Liao and Elsayed, 2006), Gamma (Tseng et al., 2009; Ling et al., 2015) and inverse Gaussian (Wang and Xu, 2010; Ye and Chen, 2013; Ye et al., 2014; Peng et al., 2014) process models mentioned in the previous Section.

The Wiener process model is often used when the degradation process is increasing or decreasing with time. If the decreasing data is discarded, Gamma or inverse Gaussian processes can be used as degradation models.

Under a unified modeling framework for ADT analysis, it is assumed that the degradation $\mathrm{X}(\mathrm{t})$ follows a stochastic process with statistically independent increments, where the mean and variance of $\mathrm{X}(\mathrm{t})$ are proportional to time. The unified stochastic process thereby defined becomes the Wiener process model when $\mathrm{X}(\mathrm{t})$ follows a normal distribution, a Gamma process when $\mathrm{X}(\mathrm{t})$ follows a Gamma distribution and an inverse Gaussian process when $\mathrm{X}(\mathrm{t})$ follows an inverse gamma distribution.

The acceleration model describes the relationship between the accelerated stress and the degradation rate. It can be obtained based on either physical knowledge of the tested equipment or empirical observations. The typical physics-based acceleration models include Arrhenius model, Eyring model, etc. (Escobar and Meeker, 2006), while the empirical acceleration models include, for example, Coffin-Manson model (Musallam et al., 2014), etc. A general log-linear form of the model can be written, whose vector of unknown parameters can be obtained by maximizing the corresponding loglikelihood functions.

Then, a $\mathrm{p}$-quantile lifetime of interest can derived from the unified stochastic process of accelerated degradation, which can be used for maintenance decision-making or verifying the lifetime and reliability levels of the tested equipment.

In practice, in $\mathrm{ADT}$ analysis, for a given dataset, more than one model might be plausible to describe it. Then, model uncertainty exists but in standard ADT data analysis, this is not yet fully considered and this may lead to wrong inferences. Actually, also in accelerated life testing (ALT), different lifetime distributions may plausibly describe the data and the problem of model uncertainty arises. This is, for example, treated in Yu and Chang (2012) by the Bayesian model averaging (BMA) 
method, with demonstration that the choice of the distribution has significant effects on the results of the lifetime evaluation at the use conditions, especially for extreme quantiles. For ADT, the Akaike's information criterion (AIC) has been introduced to select the appropriate model (Park and Padgett, 2006; Park and Padgett, 2005). However, the effect of model uncertainty on the lifetime evaluation results is not considered. In Pan and Balakrishnan (2010), both Wiener and Gamma process models have been used, and shown to give accurate parameters estimates. However, the question remains to how the degradation model affects the lifetime evaluation results and how the model uncertainty can be accounted for.

As to the acceleration models, arguments are given in $\mathrm{Yu}$ and Chang (2012) for it not to be of concern for the model uncertainty issue, since one can choose it based on physical considerations.

With respect to model uncertainty in literature, many works have addressed this issue (Laskey, 1996; Nislsen and Aven, 2003; Aven and Zio, 2011; Liu et al., 2013). In the work of Zio and Apostolakis (1996), two approaches, i.e. alternate-hypotheses (also known as model averaging) and adjustmentfactor, have been used to treat model uncertainty by expert judgments. The former one combines all the available models through a mixture of probabilities. The latter selects a best model as reference and updates it with information from the other models. Model averaging has been fully extended into Bayesian model averaging (Hoeting et al., 1999; Park and Grandhi, 2014), by the integration of model prior knowledge and the likelihood function of the obtained data for each model. In Droguett and Mosleh (2008), it is applied to account for model uncertainty based on differences between experimental observations and model predictions. One problem of the model averaging method is that it assumes that the real model is one of the candidate models since the summation of model probabilities must be equal to one, even though it is unknown and of difficult interpretation, and controversial in practical applications. To relax this assumption, Dempster-Shafer (D-S) theory can be introduced, using belief and plausibility functions to account for model uncertainty (Baraldi and Zio, 2010; Park and Granhi, 2012). Some difficulties may arise in this method, in the elicitation of expert knowledge on the belief values to assign to the models. As to the adjustment-factor method, some work has been done for its application to accelerated testing models based on field lifetime or degradation data. For example, Pan (2009) chose exponential and Weibull lifetime distributions as the reference models for Device-A ALT data; then, a calibration factor is introduced to update the reference models with the field failure data since the lab-test environment and field conditions are different. A similar procedure is used for ADT models in (Wang et al., 2013).

\section{Prognostics and Health Management}


Prognostics and Health Management (PHM) is a field of research and application aiming at detecting the degradation of engineering components, diagnosing the type of faults, predicting the failure times and proactively managing their failures.

In the last ten years, it has drawn great attention from both the research and practice points of view, because of the increased capacity in monitoring and the significant advancements in the techniques of signal and data analysis, including data mining and artificial intelligence, which enable the intelligent reading of the recorded signals and data for fault detection and diagnostics, and failure prediction.

The objective of PHM is to take past, present and (predicted) future information on the conditions of engineering components and systems, and use it for detecting their degradation, diagnosing their faults, predicting their future health state evolution and their Remaining Useful Life (RUL, namely how much time the engineering component or system can keep performing its intended function). Ideally, the accurate prediction of the future evolution of the component health state allows running it as long as it is healthy, with the possibility of planning maintenance work at the most convenient and inexpensive time. Thus, expected benefits of PHM are increased plant reliability and availability, increased equipment lifetime, increased plant safety, fewer accidents with negative impact on environment, and optimized spare parts handling (Zio, 2012).

Traditional PHM methods have been firstly developed and verified considering single engineering components and systems operating in fixed and stationary working conditions. For example, several diagnostic methods have been developed and successfully applied to bearings working at fixed and constant rotating speed and load, and prognostic methods are available for the prediction of the RUL of structures operating under constant loads at constant temperatures (Wu et al., 2012; Zhou et al., 2012). However, in practical industrial applications, there are a lot of engineering systems operating in an Evolving Environment (EE), characterized by continuous or periodic variations of the working conditions. For example, bearings in automotive vehicles are subject to continuous variation of the rotating speed and several structures work under variable loads. The main issue of applying traditional PHM in an EE is that the information available at the time of developing the PHM models usually does not cover all the possible future working conditions that the engineering components and systems will experience during their life. Thus, the traditional PHM approaches, where the predictive models are developed using information collected in a limited set of working conditions, tend to provide unsatisfactory performances when applied in the realistic EE conditions encountered in practice.

The problem of developing models able to provide satisfactory performances in presence of an EE is encountered in several research fields such as network monitoring, web mining, telecommunications and financial data management (Dyer et al., 2014). Most engineering components and systems are 
operating in an EE. For example, bearings, gears, alternators, shafts and pumps in automotive vehicles typically work in continuously varying conditions of loads (Zhao et al., 2015; Bian et al., 2013; Liu et al., 2014). Thus, in order to avoid a remarkable reduction of the performance of the PHM models when they are used in an EE, it is fundamental to develop PHM models able to continuously and automatically update themselves.

From a statistical point of view, data collected in an evolving environment are affected by a concept drift, i.e., the statistical properties of the data change over time in an unforeseen way (Elwell and Polikar, 2014). Since the relationship between model input and output tends to continuously change, learning a model in presence of a concept drift is a difficult task. A possible approach to this problem is based on the following two steps:

i. detection of the occurrence of the concept drift;

ii. once the concept drift is detected, updating the model using new input-output data collected in the new environment.

With respect to i), the task of concept drift detection is similar to that of fault detection, i.e. verifying whether there is a significant difference of the statistical properties between current and past signal values. Thus, typical residual-based approaches to fault detection such as Sequential Probability Ratio Test (SPRT) and Auto-Associative Kernel Regression (AAKR) can be applied also to the problem of concept drift detection.

With respect to ii), some algorithms able to online update the models in order to adapt them to the EE have been developed (Dries and Ruckert, 2009). Algorithms based on the ideas of domain adaptation and semi-supervised learning are capable to extract information from the drifted data, whereas active learning algorithms allow reducing the retraining cost by selecting the most informative patterns in the drifted data (Zliobaite et al., 2011). In Razavi-Far et al. (2012), an ensemble approach is proposed, where a new model is developed and added to the ensemble each time a new set of data becomes available.

With respect to the problem of fault detection in an EE, the standard methods may provide unsatisfactory performances because modifications of the working conditions can be confused with abnormal conditions, since they may have similar effects on the behavior of the measured signals. Thus, fault detection systems can provide several false alarms when they are used in an EE. For this reason, the current industrial practice is to periodically update the fault detection systems in order to limit the effects of the EE.

In the presence of an EE, empirical diagnostic models like K-Nearest Neighbors (KNN), Artificial Neural Networks (ANN), Support Vector Machines (SVM) and Relevance Vector Machines (RVM) may suffer a reduction of diagnostic performance. As mentioned earlier, the main reason is that the data used to develop them are collected in a limited set of working conditions, not sufficiently 
covering all the possible working conditions that may be experienced by the engineering system during its life. Thus, diagnostic models need to learn the modification of the mapping between the measured signals and the fault type caused by the EE. Notice that many methods of fault diagnostics in an $\mathrm{EE}$ are supervised and, thus, require the availability of input-output labeled data, i.e. the knowledge of the signal values and the corresponding fault class, whereas, in practice, the identification of the class of the fault causing the malfunctioning in an EE is often not feasible, or very expensive and time consuming. Thus, novel drift learning algorithms which do not rely on labeled data are strongly needed for fault diagnostics in an EE.

With respect to the problem of prognostics in an EE, many studies have adopted approaches based on filter algorithms, such as the Kalman and Particle Filters, which model the effect of the EE on the equipment degradation as a process noise. In practice, the degradation measurements which progressively become available are used to estimate the engineering system degradation state and its future evolution, taking into account the effect of measurement and process noises. However, these approaches are difficult to apply in situations characterized by scarce information on the degradation process, since they require the knowledge of a physics-based degradation model and its parameters. A further problem is that the presence of an EE can cause major modifications on the signal behavior which cannot be described by a process noise.

A further challenge for PHM methods is their scaling up to the fleet-level needed by the modern complex systems, comprising a large number of different components and integrating their functionalities. Indeed, the currently proposed approaches mainly consider component- or systemlevel prognostics. In such settings for PHM, it can be expected that detections, diagnostics and predictions with different degrees of uncertainty need to be aggregated for optimal maintenance decisions under various constraints, including resources and availability. For such distributed maintenance management problems, some approaches are emerging, with a formulation of the problem as multi-agent (Lei and Zuo, 2009) or holonic systems (Mavromatidi et al., 2013), but limiting to the integration and exchange of information among the agents, without further interaction and cooperation on the decision making for maintenance action, taking into account the different levels of uncertainty of the different components detections, diagnostics and predictions. On the contrary, a truly effective, distributed intelligent dynamic maintenance management system based on PHM must be capable of accommodating exchange of information, aggregation, interaction and cooperation at different levels of the system, coping with the different levels of uncertainty therein. In such maintenance management system, the operating experience from different systems within one fleet needs to be integrated in the learning process of all similar systems, looking for similarity of behavior to actually exploit differences and therefore learn the unexpected from one system to another. However, this is not a simple task to do as the operating and environmental conditions are different from one system to another in the fleet, and the patterns of evolution will not, thus, be 
directly transferable to any specific system. For this reason, the solution to the problem must be dynamic, with coordination and organization of the information exchange among the different algorithms and models tailored to the specific operating and environmental conditions of the different systems.

From the above discussion, the challenge arises of implementing a distributed intelligent dynamic maintenance management system that integrates aggregation and cooperation at different system levels, and is capable of handling different degrees of uncertainty under realistic constraints and changing operating and environmental conditions (EE). For this:

- agents at different system levels must be defined, characterized by their RUL, the associated uncertainty, the required maintenance actions and the associated costs, and their criticality with respect to the functionality and the safety of the entire system;

- different dynamics of the progression of the occurring degradation or fault must be included in the model, to account for the different EE which can be gradual or sudden/shock-like;

- the optimization problem must be formulated so as to ensure allowing that the objectives of the agents at different system levels are achieved, while also satisfying the overall system objectives and constraints.

From the industry point of view, for the practical implementation of any PHM-based maintenance policy, be it a Condition-Based Maintenance (CBM) or a Preventive Maintenance (PrM) one, two strategic problems need to be addressed:

- evaluation of the opportunity of adopting such advanced maintenance policies, founded on specialized knowledge and modern technology, to improve performance compared to the performance of 'traditional' Corrective Maintenance (CM) and PrM policies (Zio and Compare, 2013);

- to choose which PHM method is best, for the system considered.

Indeed, any PHM approach has its advantages, limitations and drawbacks, so that choosing the best approach for a given problem may be difficult. Criteria are needed to help the manager selecting the best approach for RUL estimation, for minimizing unscheduled shutdowns, maximizing availability, minimizing maintenance costs. A well-known criterion to guide design, operation and maintenance choices is the return on investment (ROI) associated to the implementation of a given PHM approach.

In literature, different methodologies have been proposed to evaluate the economic performance of a PHM approach. The indicator for evaluating the economic benefit of a PHM approach proposed in Feldman et al. (2009) is the ROI, calculated as follows:

$$
R O I=\frac{\text { Avoided cost }}{\text { Investment }}-1
$$


In the equation, the avoided cost quantifies the economic benefit realized through using PHM, while the investment cost includes all the costs related to PHM installation, support and maintenance. Usually, the ROI of a PHM approach is calculated relative to unscheduled maintenance. In this case, the avoided cost will be equivalent to the difference between the total life cycle cost of the equipment under unscheduled maintenance and the total life cycle cost of the equipment using a particular PHM approach (Si et al., 2011), as shown in equation (2):

$$
\text { Avoided cost }=C_{u s}-\left(C_{P H M}-I_{P H M}\right)
$$

where $C_{u s}$ is the life cycle cost in case of unscheduled maintenance, $C_{P H M}$ is the life cycle cost in case of adopting PHM and $I_{P H M}$ is the investment cost of PHM implementation.

By using the expression of the avoided cost in equation (2), the ROI of a PHM approach in equation (1) becomes:

$$
R O I=\frac{C_{u S}-\left(C_{P H M}-I_{P H M}\right)}{I_{P H M}}-1
$$

Depending on the value of ROI, the decision maker can choose whether to implement the PHM approach or not: if the ROI is positive, then it is recommended to implement the PHM approach; if the ROI is null or negative, there is no benefit for implementing PHM and an unscheduled maintenance strategy would be preferable because, different to the predictive maintenance strategy, unscheduled maintenance does not require additional costs related to implementation.

Indeed, ROI analysis may be used for different purposes: selection of the best PHM approach to implement, optimization of the setting and use of a particular PHM approach, and determination of whether one should adopt PHM and predictive maintenance at all or another type of maintenance (Feldman et al., 2009). Some authors have discussed in the literature the economic justification of PHM (Feldman et al., 2008; Sandborn and Wilkinson, 2007; Zhang, 2013; Leao et al., 2008; Nilsson et al., 2007; Chang et al., 2015; Fritzsche et al., 2014; Pecht, 2012). For example, the use of ROI for PHM in malfunctioned displays of Boeing 737 has been presented in Feldman et al. (2008). PHM results of linear replaceable units have been used to define maintenance policies and take related decisions aimed at minimizing life cycle costs or maximizing availability in Sandborn and Wilkinson (2007). Applications considering ROI for PHM of medical devices and commercial aircrafts have been reported in Zhang (2013) and Leao et al. (2008). More specifically, the cost-benefit analysis of PHM in the field of medical devices discussed in Zhang (2013) shows that the implementation of PHM in medicine-dispensing products can save $36.2 \%$ of the service cost for the installed products in eight cities (Zhang, 2013), and the methodology developed in Leao et al. (2008) evaluates the economic benefits of PHM for application to commercial aircrafts considering four categories: benefits of monitoring the system, benefits of prognostics, benefits of complete health management 
and intangible benefits; for each type of benefit, a mathematical model is developed to quantify the economic value of the benefit. In a second step and with the same logic, the costs of PHM are categorized into three categories: development costs, aircraft costs and costs of PHM side effects, with associated mathematical models for their quantification.

Life-cycle cost analysis has been performed in Nilsson et al. (2007) to improve maintenance planning for a single wind turbine onshore and a wind farm offshore. The life cycle cost is calculated for both unscheduled and scheduled maintenance as the sum of the cost of investment, the cost of corrective and preventive maintenance, the cost for production loss and the cost of the remainder value. The results show that for an entire farm of wind power systems, CBM is the most profitable and a simple increase on the availability of the system $(0.43 \%)$ obtained by the use of a Condition Monitoring System (CMS) is sufficient to cover the costs related to implementing the CMS itself.

System monitoring and ROI analysis have been combined in Chang et al. (2015) for maintenance planning of LED lighting systems. Prognostics has been introduced in Fritzsche et al. (2014) to minimize maintenance costs in the airline industry.

However, the existing approaches and frameworks used to evaluate the ROI of a PHM approach suffer from many gaps:

- they are for specific applications: they are not general and only fit the case study in which they are applied (Feldman et al., 2009);

- they do not consider the impact of the performance of a PHM approach on the economic benefit generated by it;

- they only provide a point value estimation of the ROI, without taking into account the uncertainties in the values of the model parameters (Feldman et al., 2009).

Thus, a more general framework to calculate the ROI of a PHM approach needs to be established, relating the ROI to different indicators of the performance of a PHM approach, which is described by characteristics of the prediction distribution obtained by it. Such framework can be used to set PHM requirements, i.e. the proper values of the PHM performance indicators that are required to achieve the ROI goal. In this view, it can provide support for managers: for a fixed minimal value (threshold) of ROI required to achieve PHM benefits, the values to be achieved by the performance indicators for obtaining this threshold can be evaluated.

In all generality, the ROI of the PHM approach is calculated by equation (1) above. The avoided cost is the gain from improvements of availability, reliability, maintainability and avoidance of failures, thanks to the PHM approach implementation. It includes the costs of failures avoided and the minimization of the loss of the RUL due to the fact that the component or system is replaced before it is fully exploited. The investment costs are the costs associated with the realization of PHM, the technologies and support necessary to integrate and incorporate PHM into the component or system. 
The value of the cost avoided in equation (1) depends on the effectiveness of the PHM approach used to predict the RUL. This depends on the degree to which the predictions are precise and reliable. With respect to this, the ability of a model to predict accurately and with confidence the RUL of a component or system has become more and more critical for the development of an appropriate, timely, and cost-benefitting maintenance scheme (Kan et al., 2015). The selection of the most appropriate PHM model for practical implementation in the field of interest relies on the possibility of the user to evaluate the prognostic performance of the available models, used in fields and applications similar to the one in question (Sirkoska et al., 2011). To quantify such prognostic performance, a set of Prognostic Performance Indicators (PPIs), simple to calculate and intuitive to understand, is needed for the comparative analysis of the different models leading to the choice of the one to be implemented (Saxena et al., 2008).

However, as of today, prognostic models lack standard definitions of their prognostic performances by widely agreed PPIs, because these latter suffer of inconsistent interpretations mainly due to varied applications and domain information (Uckun et al., 2008; Sharp, 2013). A common approach to define the PPIs of a model needs to be developed, starting from the PPIs that have been proposed in literature for a variety of field applications.

For evaluating the capabilities of prognostic models, by PPIs quantification, it is useful to classify the prognostic models in RUL-based and degradation-based models. Despite that for both classes of models the final goal is the estimation of the RUL they, however, differ in the process of determining the RUL, as in the first case it is calculated directly from the raw data, whereas in the second case it is calculated indirectly on the basis of a Health Index (HI), constructed based on raw data (Saxena et al., 2014). In other words, in the first case, the raw data is processed and used directly for prognostic purposes in the RUL-based model; in the second case, the raw data is manipulated to obtain a HI, which is a quantifiable characteristic of the equipment of interest that aggregates the relevant features and operational conditions identifying its health (Saxena et al., 2008). The HI is, then, used in a degradation-based model, to retrieve the RUL of the equipment.

Whilst for the RUL-based models, their prognostic performance can be directly quantified by looking for the estimated RUL characteristics, for the degradation-based models, the prognostic performance is dependent also on the HI characteristics. In fact, for a HI to be useful for RUL prediction, it must have certain features and characteristics, such as being monotonic and with defined trend. After the HI is assessed to be suitable for being used for RUL prediction, the degradation-based models can be used and their PPIs coherently quantified.

Based on these premises, different classes of PPIs exist: PPIs for RUL performance quantification and for HI performance quantification. Both PPIs for RUL and HI can manipulate either instantaneous information at a given instant $t$ or the integral information from the moment of interest to the end of the predicted life. 
Irrespective of the instantaneous or integral manipulation of the available information, the PPIs can be primarily classified according to their characteristics: i) Accuracy, ii) Precision, iii) Stability and iv) Spill-Over.

Accuracy PPIs quantify the closeness between the model output and the true value (Saxena et al., 2014; Saxena et al., 2008; Walther and Moore, 2005). A very accurate model will have an estimated RUL very close to the true one.

Precision PPIs measure the spread of the model output, by measuring the statistical variance and the spread of the data attributable to statistical variability (Saxena et al., 2014; Walther and Moore, 2005). They quantify how confident the model is on the estimate and the degree to which a repetition of the prognosis will yield the same results.

Stability PPIs quantify the models sensitivity by evaluating the models output with respect to factors that directly affect the RUL estimation and prediction (i.e., the models input), and assess the models ability to, in the long run, tend to the correct value, i.e., converge (Johnson et al., 2011; Saxena et al., 2014). The stability of a model allows for an increased confidence in the models output.

Spill-Over PPIs measure the effects of varying the inputs on the models performance, by evaluating the effects of reducing the number of features of a model (Saxena et al., 2008).

To compare the performances of various prognostic models, one would need to resort to a PPIs aggregation technique, which would allow for a quantitative overall assessment of the prognostic performances. The aggregation technique must enable the analyst to intuitively grasp the meaning of the results provided by the PPIs and must deliver a clear suggestion for the prognostic model to be chosen among the available ones.

For the purposes of sketching the idea of a ROI-based framework of evaluation, we consider the case where the performance of the PHM approach is measured by the Precision Index (PI), which describes how close the predictions are clustered together and it is a measure of the narrowness of the interval in which the RUL value is expected to really fall (the smaller the PI is, the more precise the estimations given by the PHM approach are) and the reliability of the PHM approach can be measured by the Risk Index (RI), defined as the probability that the RUL predicted by PHM is lower than the threshold for preventive maintenance time $T_{h}$. Mathematically, the $R I$ will be equal to the area below the RUL distribution curve, for $R U L<T_{h}$. The higher the RI is, the less reliable the implemented PHM is.

The cost of loss of the RUL of the component or system, $(C L)$, depends on the difference between the time of replacement of the component or system and its actual time of failure. This cost is higher, the earlier the component or system is replaced. A threshold is given by the manager as the minimal time needed for maintenance and $C L$ can be calculated as the integral of the RUL prediction distribution between the threshold and the mean value of the prediction, multiplied by the cost of the remaining useful life per unit of time. 
The cost of corrective maintenance $(C M)$ and the cost of failures avoided $(F A)$ are calculated with respect to the case when the RUL predicted by PHM exceeds $T_{h}$. In this sense, the avoided cost depends on the risk index. Given the avoided cost, the ROI of a PHM approach can be calculated and a relationship can be obtained between the ROI and the characteristics of the predicted $R U L$ distribution.

A framework such as the one sketched above can be used to guide the selection of the adequate PHM approach to implement in order to reach a desired ROI. In fact, the decision maker can define the requirements for the performance metrics of the PHM approach leading to a minimal desired ROI. It is expected that to reach a high value of ROI, the predictions should be precise and the component or system should be repaired/replaced at a time such to avoid the failure of the equipment on one hand but, also, minimize, on the other hand, the costs related to the loss of still exploitable RUL. Note that since the predictions of PHM are subject to uncertainties, the framework of ROI evaluation should be able to include the changing operational and environmental factors that may affect the RUL predictions. Moreover, in practice also, the threshold for preventive maintenance time $T_{h}$ is typically uncertain and, thus, sensitivity analysis should be performed to study how the variation of $T_{h}$ affects the variation of the ROI of a given PHM approach.

The ROI-based evaluation framework sketched above is only illustrative and, in practice, to ensure that the adopted PHM method meets the goal of confident maintenance decision making, the prognostic method upon which to rely for RUL prediction should not only satisfy the requirements on the expected quality of the RUL point estimate (e.g., the accuracy PPIs described), on uncertainty quantification (e.g., the precision PPIs described above), and on the other desirable characteristics (measured by the other PPIs mentioned above), but also meet some requirements of trustworthiness of the prognostic method itself, i.e., the credibility that for the specific RUL prediction problem of interest, the method can be trusted to provide an accurate and precise RUL with correct and fair uncertainty quantification.

In this view, one must assess the prediction capability of a prognostic method, i.e. the property of providing trustable RUL predictions, with the quality characteristics required for the purpose of the maintenance decision making of interest. The RUL quality characteristics are measured by the PPIs defined above. For the trustworthiness of the prognostic method, one must introduce some concept of prediction capability maturity to measure the property that a prognostic method can provide trustworthy results, based on the inherent characteristics of the method itself in relation to RUL predictions, and on proven experiences of application in other problem settings similar to the one under analysis: the more mature a prognostic method, the more confidence that its predictions can be trusted.

To evaluate the prediction capability of a prognostic method, both the $R U L$ prediction quality (as measured by the PPIs) and the trustworthiness of the prognostic method itself should be considered, 
as illustrated in Figure 1Figure 1. As seen above, a number of approaches can be used for measuring the quality performance of a prognostic method by evaluating its RUL predictions with respect to whether the method can yield accurate and precise RUL estimates, considering both point estimate and uncertainty (i.e., in terms of the PPIs). On the other hand, only few methods have been proposed for the assessment of the trustworthiness of a prediction method or model (Paulk et al., 1993; Oberkampf et al., 2007), and none exists in the specific context and for the specific aim of prognostics and maintenance decision making. To fill this gap, a structured framework should be proposed for the assessment of the prediction capability maturity of a prognostics method, to be integrated with the RUL prediction quality, measured conditioned on the prognostic model performance given the available data, to yield the overall prognostic method prediction capability (Higure 1Figure 1). 


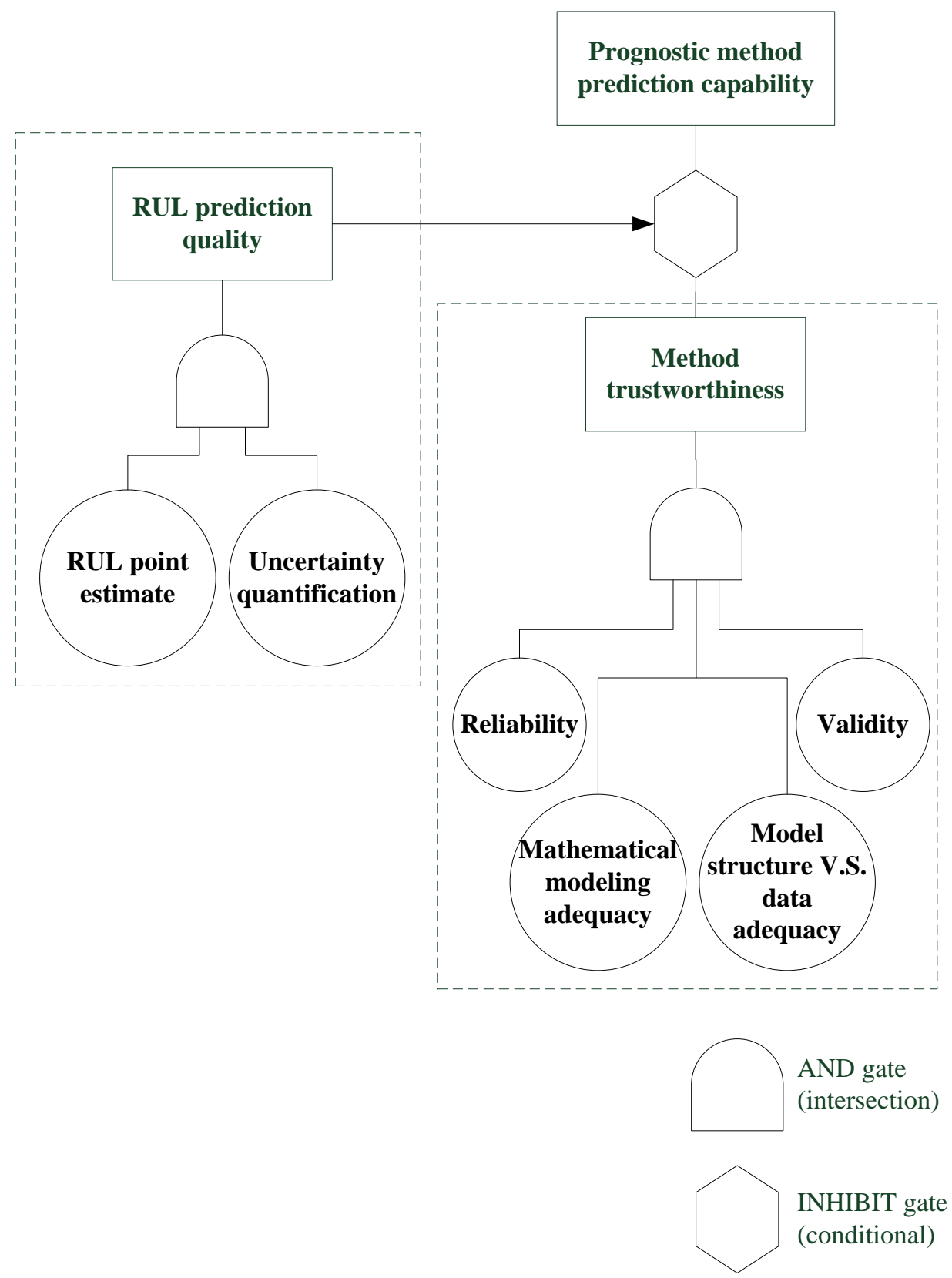

Figure 1 Prediction capability consists of RUL prediction quality and prognostic method trustworthiness. The former can be quantified resorting to the Prognostic Performance Indicators (PPIs), whereas the latter should be quantified resorting to a structured prediction capability maturity assessment framework.

Finally, PHM utilizing databases and information technology is the enabling technique for condition-based and predictive maintenance. However, in practice a challenge for the use of PHM, and the associated condition-based and predictive maintenance policies, comes to the reliability qualification of a component or system at the design stage, for its licensing. The 
question is how to include these aspects in the reliability qualification or safety case: how must these be changed when scheduled maintenance is replaced with condition-based and/or predictive maintenance? This question has become fundamental, as industry wishes to move to these more confident, effective, flexible, smart maintenance policies of their smart components and systems, but safety and reliability demonstration are still needed and required. The PHM techniques using condition monitoring data can contribute to the analysis for evaluating failure frequency and probability with reduced conservatism, thus allowing for an online reliability assessment, with reduced uncertainty. In the online, living assessment, if the statistics-based distributions are replaced with condition-based distributions obtained by PHM reliability estimates and uncertainty bands are no longer fixed a priori but change depending on the predictive power of the PHM techniques employed. The frequency of failures, the equipment unavailability, the probability of human error and the probability of recovery a failure change due to aging effects and other environmental and operational factors, the components and systems reliability estimates change too, and so do the associated uncertainty bands. This requires that the estimation be done time-dependently on the basis of the prognostics results, based on the prior assessment and a Bayesian update using the prognostics data (Kim et al., 2015).

\section{Conclusions}

The reliability engineering field must continuously evolve to be at speed with the industrial and societal developments. This requires continuous advancement of technical knowledge and ability. In the current scenario of technological development, with strong digitalization and interconnection at all levels of cyber-physical systems and in all industrial sectors, reliability engineering is faced with new challenges but it is also exposed to new opportunities of improvement.

In this paper, I have focused on the reliability modeling of components and systems, and discussed some challenges and opportunities related to degradation modeling and prognostics and health management. These areas of research and application hold great promise for the improvement of the safety and the productive and service capacity of industrial components and systems. The knowledge, information and data that are increasingly acquired offer new ways of modeling and analysis, with significant potential benefits.

The practical implementation of these new ways needs to be followed up with care, for effectively collecting such benefits. For example, it is clear that PHM utilizing databases and information technology is the enabling technique for condition-based and predictive maintenance. However, in practice a challenge for the use of PHM, and the associated condition-based and predictive maintenance policies, comes to the reliability qualification of a component or system that must be done a priori, at the design stage, for its licensing. The question is how to include these aspects in the reliability qualification or safety case: how must these be changed when scheduled maintenance is replaced with condition-based and/or predictive maintenance? This question has become fundamental, as industry wishes to move to these more confident, effective, flexible, smart maintenance policies of their smart 
components and systems, but safety and reliability demonstration are still needed and required.

Furthermore, the PHM techniques using condition monitoring data can contribute to the analysis for evaluating failure frequency and probability with reduced conservatism, thus allowing for an online reliability assessment, with reduced uncertainty. In the online, living assessment, if the statistics-based distributions are replaced with condition-based distributions obtained by PHM, reliability estimates and uncertainty bands are no longer fixed a priori but change depending on the predictive power of the PHM techniques employed. As the frequency of failures, the equipment unavailability, the probability of human error and the probability of recovering a failure change due to aging effects and other environmental and operational factors, the components and systems reliability estimates change too, and so do the associated uncertainty bands. This requires that the estimation be done time-dependently on the basis of the prognostics results, based on a prior assessment and a Bayesian update using the prognostics data (Kim et al., 2015).

\section{Acknowledgments}

I would like to acknowledge the insightful work and sharing experience of the many colleagues which have shared with me passionate study and research experiences on the subjects of this paper. These are the colleagues from the Chaire on Systems Science and the Energetic Challenge (SSEC) of CentraleSupelec, Paris, www.ssde.fr , the Laboratory of Signal Analysis and Risk Analysis (LASAR) at the Department of Energy of the Politecnico di Milano, www.lasar.polimi.it, the Center for Resilience and Safety of Critical Infrastructures (CRESCI), http://cresci.buaa.edu.cn/, and Prof. Olga Fink. Deep thanks go also to Dr. Jie Liu of CentraleSupelec, Paris, for his contribution in the preparation of the manuscript.

\section{References}

Agrafiotis, G. K., and M. Z. Tsoukalas. "On excess-time correlated cumulative processes." Journal of the Operational Research Society (1995): 1269-1280.

Alam, Mansoor, and Ubaid M. Al-Saggaf. "Quantitative reliability evaluation of repairable phasedmission systems using Markov approach." IEEE Transactions on Reliability 5.35 (1986): 498503.

Albin, S. L., and Seng Chao. "Preventive replacement in systems with dependent components." Reliability, IEEE Transactions on 41.2 (1992): 230-238.

Anderson, Kevin K. "Limit theorems for general shock models with infinite mean intershock times." Journal of applied probability (1987): 449-456.

Aven, Terje, and Enrico Zio. "Some considerations on the treatment of uncertainties in risk assessment for practical decision making." Reliability Engineering \& System Safety 96.1 (2011): 64-74.

Bai, Jian-Ming, Ze-Hui Li, and Xin-Bing Kong. "Generalized shock models based on a cluster point process." Reliability, IEEE Transactions on 55.3 (2006): 542-550. 
Baraldi, Piero, and Enrico Zio. "A Comparison Between Probabilistic and Dempster-Shafer Theory Approaches to Model Uncertainty Analysis in the Performance Assessment of Radioactive Waste Repositories." Risk Analysis 30.7 (2010): 1139-1156.

Barros, Anne, Christophe Berenguer, and Antoine Grall. "A maintenance policy for two-unit parallel systems based on imperfect monitoring information." Reliability Engineering \& System Safety 91.2 (2006): 131-136.

Becker, Günter, Leonidas Camarinopoulos, and Dimitris Kabranis. "Dynamic reliability under random shocks." Reliability Engineering \& System Safety 77.3 (2002): 239-251.

Bian, Linkan, and Nagi Gebraeel. "Stochastic methodology for prognostics under continuously varying environmental profiles." Statistical Analysis and Data Mining 6.3 (2013): 260-270.

Black, M., A. T. Brint, and J. R. Brailsford. "A semi-Markov approach for modelling asset deterioration." Journal of the Operational Research Society 56.11 (2005): 1241-1249.

Bondavalli, Andrea, et al. "Dependability modeling and evaluation of multiple-phased systems using DEEM." Reliability, IEEE Transactions on 53.4 (2004): 509-522.

Castanier, Bruno, Antoine Grall, and Christophe Bérenguer. "A condition-based maintenance policy with non-periodic inspections for a two-unit series system." Reliability Engineering \& System Safety 87.1 (2005): 109-120.

Chalk, Steven G., and James F. Miller. "Key challenges and recent progress in batteries, fuel cells, and hydrogen storage for clean energy systems." Journal of Power Sources 159.1 (2006): 7380.

Chang, Moon-Hwan, et al. "A return on investment analysis of applying health monitoring to LED lighting systems." Microelectronics Reliability 55.3 (2015): 527-537.

Chen, Nan, et al. "Condition-based maintenance using the inverse Gaussian degradation model." European Journal of Operational Research 243.1 (2015): 190-199.

Chookah, Mohamed, Mohammad Nuhi, and Mohammad Modarres. "A probabilistic physics-of-failure model for prognostic health management of structures subject to pitting and corrosion-fatigue." Reliability Engineering \& System Safety 96.12 (2011): 1601-1610.

Daigle, Matthew J., and Kai Goebel. "A model-based prognostics approach applied to pneumatic valves." International Journal of Prognostics and Health Management 2 (2011): 84.

Liu, Dong, et al. "Reliability analysis of phased-mission systems using Bayesian networks." Reliability and Maintainability Symposium, 2008. RAMS 2008. Annual. IEEE, 2008.

Dekker, Rommert, Raymond Ph Plasmeijer, and Jan H. Swart. "Evaluation of a new maintenance concept for the preservation of highways." IMA Journal of Management Mathematics 9.2 (1998): 109-156.

Dries, Anton, and Ulrich Rückert. "Adaptive concept drift detection." Statistical Analysis and Data Mining 2.5-6 (2009): 311-327.

Droguett, Enrique López, and Ali Mosleh. "Bayesian methodology for model uncertainty using model performance data." Risk Analysis 28.5 (2008): 1457-1476.

Dugan, JoAnne Bechta. "Automated analysis of phased-mission reliability." Reliability, IEEE Transactions on 40.1 (1991): 45-52.

Dyer, Karl B., Robert Capo, and Robi Polikar. "Compose: A semisupervised learning framework for initially labeled nonstationary streaming data." Neural Networks and Learning Systems, IEEE Transactions on 25.1 (2014): 12-26.

Elwell, Ryan, and Robi Polikar. "Incremental learning of concept drift in nonstationary environments." Neural Networks, IEEE Transactions on 22.10 (2011): 1517-1531.

Esary, J. D., and A. W. Marshall. "Shock models and wear processes." The annals of probability (1973): 627-649.

Escobar, Luis A., and William Q. Meeker. "A review of accelerated test models." Statistical Science (2006): 552-577. 
Fan, Juanjuan, S. G. Ghurye, and Richard A. Levine. "Multicomponent lifetime distributions in the presence of ageing." Journal of applied probability 37.2 (2000): 521-533.

Feldman, Kiri, Peter Sandborn, and Taoufik Jazouli. "The analysis of return on investment for PHM applied to electronic systems." Prognostics and Health Management, 2008. PHM 2008. International Conference on. IEEE, 2008.

Feldman, Kiri, Taoufik Jazouli, and Peter A. Sandborn. "A methodology for determining the return on investment associated with prognostics and health management." Reliability, IEEE Transactions on 58.2 (2009): 305-316.

Fritzsche, R., J. N. D. Gupta, and R. Lasch. "Optimal prognostic distance to minimize total maintenance cost: The case of the airline industry." International Journal of Production Economics 151 (2014): 76-88.

Fussel J. B., et al. "Phased-Mission System Reliability Analysis," Volume 1: Methodology. Electric Power Research Institute Research Report 1233-2. University of Tennessee, Knoxville Tennessee, July 1981.

Gebraeel, Nagi, Alaa Elwany, and Jing Pan. "Residual life predictions in the absence of prior degradation knowledge." Reliability, IEEE Transactions on 58.1 (2009): 106-117.

Giorgio, Massimiliano, Maurizio Guida, and Gianpaolo Pulcini. "An age-and state-dependent Markov model for degradation processes." IIE Transactions 43.9 (2011): 621-632.

Hoeting, Jennifer A., et al. "Bayesian model averaging: a tutorial." Statistical science (1999): 382-401.

Hong, H. P., et al. "Optimal condition-based maintenance decisions for systems with dependent stochastic degradation of components." Reliability Engineering \& System Safety 121 (2014): 276-288.

Kim, Hyeonmin, et al. "Reliability data update using condition monitoring and prognostics in probabilistic safety assessment." Nuclear Engineering and Technology 47.2 (2015): 204-211.

Jiang, Lei, Qianmei Feng, and David W. Coit. "Reliability and maintenance modeling for dependent competing failure processes with shifting failure thresholds." Reliability, IEEE Transactions on 61.4 (2012): 932-948.

Johnson, Stephen B., et al., eds. System health management: with aerospace applications. John Wiley \& Sons, 2011.

Kan, Man Shan, Andy CC Tan, and Joseph Mathew. "A review on prognostic techniques for nonstationary and non-linear rotating systems." Mechanical Systems and Signal Processing 62 (2015): 1-20.

Keedy, Elias, and Qianmei Feng. "A physics-of-failure based reliability and maintenance modeling framework for stent deployment and operation." Reliability Engineering \& System Safety 103 (2012): 94-101.

Klutke, Georgia-Ann, and Yoonjung Yang. "The availability of inspected systems subject to shocks and graceful degradation." Reliability, IEEE Transactions on 51.3 (2002): 371-374.

Kim, Kuk, and Kyung S. Park. "Phased-mission system reliability under Markov environment." Reliability, IEEE Transactions on 43.2 (1994): 301-309.

Kröger, Wolfgang, and Enrico Zio. Vulnerable systems. Springer Science \& Business Media, 2011.

Lai, Min-Tsai, and Ying-Chang Chen. "Optimal periodic replacement policy for a two-unit system with failure rate interaction." The international journal of advanced manufacturing technology 29.34 (2006): 367-371.

Laskey, Kathryn Blackmond. "Model uncertainty: Theory and practical implications." Systems, Man and Cybernetics, Part A: Systems and Humans, IEEE Transactions on 26.3 (1996): 340-348.

Lawless, Jerry, and Martin Crowder. "Covariates and random effects in a gamma process model with application to degradation and failure." Lifetime Data Analysis 10.3 (2004): 213-227.

Leão, Bruno P., et al. "Cost-benefit analysis methodology for PHM applied to legacy commercial aircraft." Aerospace Conference, 2008 IEEE. IEEE, 2008. 
Lei, Yaguo, and Ming J. Zuo. "Gear crack level identification based on weighted K nearest neighbor classification algorithm." Mechanical Systems and Signal Processing 23.5 (2009): 1535-1547.

$\mathrm{Li}$, Wenjian, and Hoang Pham. "Reliability modeling of multi-state degraded systems with multicompeting failures and random shocks." Reliability, IEEE Transactions on 54.2 (2005): 297303.

Li, Yan-Fu, Enrico Zio, and Yan-Hui Lin. "A multistate physics model of component degradation based on stochastic petri nets and simulation." Reliability, IEEE Transactions on 61.4 (2012): 921931.

Liao, Haitao, and Elsayed A. Elsayed. "Reliability inference for field conditions from accelerated degradation testing." Naval Research Logistics (NRL) 53.6 (2006): 576-587.

Levitin, G., and A. Lisnianski. "Multi-state system reliability: assessment, optimization and applications." (2003).

Lim, Heonsang, and Bong-Jin Yum. "Optimal design of accelerated degradation tests based on Wiener process models." Journal of Applied Statistics 38.2 (2011): 309-325.

Lin, Yan-Hui, Yan-Fu Li, and Enrico Zio. "Fuzzy reliability assessment of systems with multipledependent competing degradation processes." Fuzzy Systems, IEEE Transactions on 23.5 (2015): 1428-1438.

Ling, Man Ho, Kwok Leung Tsui, and Narayanaswamy Balakrishnan. "Accelerated degradation analysis for the quality of a system based on the Gamma process." Reliability, IEEE Transactions on 64.1 (2015): 463-472.

Liu, Jie, et al. "Nuclear power plant components condition monitoring by probabilistic support vector machine." Annals of Nuclear Energy 56 (2013): 23-33.

Liu, Jie, et al. "AN EFFICIENT ONLINE LEARNING APPROACH FOR SUPPORT VECTOR REGRESSION." Decision Making and Soft Computing: Proceedings of the 11th International FLINS Conference. Vol. 10. World Scientific, 2014.

Lu, C. Joseph, and William O. Meeker. "Using degradation measures to estimate a time-to-failure distribution." Technometrics 35.2 (1993): 161-174.

Lu, Jye-Chyi, Jinho Park, and Qing Yang. "Statistical inference of a time-to-failure distribution derived from linear degradation data." Technometrics 39.4 (1997): 391-400.

Lydell, Bengt OY. "Pipe failure probability—the Thomas paper revisited." Reliability Engineering \& System Safety 68.3 (2000): 207-217.

Mavromatidis, Georgios, Salvador Acha, and Nilay Shah. "Diagnostic tools of energy performance for supermarkets using Artificial Neural Network algorithms." Energy and Buildings 62 (2013): 304-314.

Mura, Ivan, and Andrea Bondavalli. "Hierarchical modeling and evaluation of phased-mission systems." Reliability, IEEE Transactions on 48.4 (1999): 360-368.

Meeker, William Q., and Luis A. Escobar. Statistical methods for reliability data. John Wiley \& Sons, 2014.

Moghaddass, Ramin, and Ming J. Zuo. "Multistate degradation and supervised estimation methods for a condition-monitored device." IIE Transactions 46.2 (2014): 131-148.

Murthy, D. N. P., and D. G. Nguyen. "Study of a multi-component system with failure interaction." European Journal of Operational Research 21.3 (1985): 330-338.

Mura, Ivan, and Andrea Bondavalli. "Markov regenerative stochastic Petri nets to model and evaluate phased mission systems dependability." IEEE Transactions on Computers 12 (2001): 13371351.

Musallam, Mahera, et al. "Application of coupled electro-thermal and physics-of-failure-based analysis to the design of accelerated life tests for power modules." Microelectronics Reliability 54.1 (2014): 172-181.

Pecht, Michael. "Nvidia's GPU failures: A case for prognostics and health management." Microelectronics Reliability 52.6 (2012): 953-957. 
Nelson, Wayne B. Accelerated testing: statistical models, test plans, and data analysis. Vol. 344. John Wiley \& Sons, 2009.

Nicolai, Robin P., and Rommert Dekker. Optimal maintenance of multi-component systems: a review. Springer London, 2008.

Nilsen, Thomas, and Terje Aven. "Models and model uncertainty in the context of risk analysis." Reliability Engineering \& System Safety 79.3 (2003): 309-317.

Nilsson, Julia, and Lina Bertling. "Maintenance management of wind power systems using condition monitoring systems-life cycle cost analysis for two case studies." Energy Conversion, IEEE Transactions on 22.1 (2007): 223-229.

Özekici, Süleyman. "Optimal periodic replacement of multicomponent reliability systems." Operations Research 36.4 (1988): 542-552.

Oberkampf, William L., Martin Pilch, and Timothy G. Trucano. Predictive capability maturity model for computational modeling and simulation. No. SAND2007-5948. Sandia National Laboratories, 2007.

Pan, Rong. "A Bayes approach to reliability prediction utilizing data from accelerated life tests and field failure observations." Quality and Reliability Engineering International 25.2 (2009): 229-240.

Pan, Zhengqiang, and N. Balakrishnan*. "Multiple-steps step-stress accelerated degradation modeling based on Wiener and gamma processes." Communications in Statistics-Simulation and Computation 39.7 (2010): 1384-1402.

Park, Chanseok, and William J. Padgett. "Stochastic degradation models with several accelerating variables." Reliability, IEEE Transactions on 55.2 (2006): 379-390.

Park, Inseok, and Ramana V. Grandhi. "Quantification of model-form and parametric uncertainty using evidence theory." Structural Safety 39 (2012): 44-51.

Park, Inseok, and Ramana V. Grandhi. "A Bayesian statistical method for quantifying model form uncertainty and two model combination methods." Reliability Engineering \& System Safety 129 (2014): 46-56.

Park, Chanseok, and W. J. Padgett. "Accelerated degradation models for failure based on geometric Brownian motion and gamma processes." Lifetime Data Analysis 11.4 (2005): 511-527.

Paulk, Mark C., et al. "Capability maturity model, version 1.1." Software, IEEE 10.4 (1993): 18-27.

Peng, Weiwen, et al. "Inverse Gaussian process models for degradation analysis: A Bayesian perspective." Reliability Engineering \& System Safety 130 (2014): 175-189.

Peng, Hao, Qianmei Feng, and David W. Coit. "Reliability and maintenance modeling for systems subject to multiple dependent competing failure processes." IIE transactions 43.1 (2010): 1222.

Rafiee, Koosha, Qianmei Feng, and David W. Coit. "Reliability modeling for dependent competing failure processes with changing degradation rate." IIE transactions 46.5 (2014): 483-496.

Rasmekomen, Nipat, and Ajith Kumar Parlikad. "Maintenance optimization for asset systems with dependent performance degradation." Reliability, IEEE Transactions on 62.2 (2013): 362-367.

Razavi-Far, Roozbeh, Piero Baraldi, and Enrico Zio. "Dynamic weighting ensembles for incremental learning and diagnosing new concept class faults in nuclear power systems." Nuclear Science, IEEE Transactions on 59.5 (2012): 2520-2530.

Reggiani, Susanna, et al. "Physics-based analytical model for HCS degradation in STI-LDMOS transistors." Electron Devices, IEEE Transactions on 58.9 (2011): 3072-3080.

Salonen, Jorma, et al. "Experience on in-service damage in power plant components." Engineering Failure Analysis 14.6 (2007): 970-977.

Sandborn, Peter A., and Chris Wilkinson. "A maintenance planning and business case development model for the application of prognostics and health management (PHM) to electronic systems." Microelectronics Reliability 47.12 (2007): 1889-1901.

Santini, Thomas, et al. "Accelerated degradation data of SiC MOSFETs for lifetime and Remaining 
Useful Life assessment." Microelectronics Reliability 54.9 (2014): 1718-1723.

Saxena, Abhinav, Shankar Sankararaman, and Kai Goebel. "Performance evaluation for fleet-based and unit-based prognostic methods." Second European conference of the Prognostics and Health Management society. 2014.

Saxena, Abhinav, et al. "Metrics for evaluating performance of prognostic techniques." Prognostics and health management, 2008. phm 2008. international conference on. IEEE, 2008.

Sharp, Michael E. "Simple Metrics for Evaluating and Conveying Prognostic Model Performance To Users With Varied Backgrounds." In other words 1 (2013): 1.

$\mathrm{Si}$, Xiao-Sheng, et al. "Remaining useful life estimation-a review on the statistical data driven approaches." European Journal of Operational Research 213.1 (2011): 1-14.

Sikorska, J. Z., Melinda Hodkiewicz, and Lin Ma. "Prognostic modelling options for remaining useful life estimation by industry." Mechanical Systems and Signal Processing 25.5 (2011): 18031836.

Smotherman, Mark, and Kay Zemoudeh. "A non-homogeneous Markov model for phased-mission reliability analysis." Reliability, IEEE Transactions on 38.5 (1989): 585-590.

Song, Sanling, et al. "Reliability analysis for multi-component systems subject to multiple dependent competing failure processes." Reliability, IEEE Transactions on 63.1 (2014): 331-345.

Song, Sanling, David W. Coit, and Qianmei Feng. "Reliability for systems of degrading components with distinct component shock sets." Reliability Engineering \& System Safety 132 (2014): 115124.

Somani, Arun K., James A. Ritcey, and Stephen HL Au. "Computationally-efficient phased-mission reliability analysis for systems with variable configurations." Reliability, IEEE Transactions on 41.4 (1992): 504-511.

Straub, Daniel. "Stochastic modeling of deterioration processes through dynamic Bayesian networks." Journal of Engineering Mechanics 135.10 (2009): 1089-1099.

Thomas, L. C. "A survey of maintenance and replacement models for maintainability and reliability of multi-item systems." Reliability Engineering 16.4 (1986): 297-309.

Thomas, E. V., et al. "Statistical methodology for predicting the life of lithium-ion cells via accelerated degradation testing." Journal of Power Sources 184.1 (2008): 312-317.

Tseng, Sheng-Tsaing, Narayanaswamy Balakrishnan, and Chih-Chun Tsai. "Optimal step-stress accelerated degradation test plan for gamma degradation processes." Reliability, IEEE Transactions on 58.4 (2009): 611-618.

Uckun, Serdar, Kai Goebel, and Peter JF Lucas. "Standardizing research methods for prognostics." Prognostics and Health Management, 2008. PHM 2008. International Conference on. IEEE, 2008.

Unwin, Stephen D., et al. Multi-state physics models of aging passive components in probabilistic risk assessment. No. PNNL-SA-76893. Pacific Northwest National Laboratory (PNNL), Richland, WA (US), 2011.

van Dijkhuizen, Gerhard. "Maintenance grouping in multi-step multi-component production systems." Maintenance, modeling and optimization. Springer US, 2000. 283-306.

Van Horenbeek, Adriaan, and Liliane Pintelon. "A dynamic predictive maintenance policy for complex multi-component systems." Reliability Engineering \& System Safety 120 (2013): 39-50.

Walther, Bruno A., and Joslin L. Moore. "The concepts of bias, precision and accuracy, and their use in testing the performance of species richness estimators, with a literature review of estimator performance." Ecography 28.6 (2005): 815-829.

Wang, Chaonan, Liudong Xing, and Gregory Levitin. "Competing failure analysis in phased-mission systems with functional dependence in one of phases." Reliability Engineering \& System Safety 108 (2012): 90-99.

Wang, Yaping, and Hoang Pham. "Modeling the dependent competing risks with multiple degradation processes and random shock using time-varying copulas." Reliability, IEEE Transactions on 
61.1 (2012): 13-22.

Wang, Xiao, and Dihua Xu. "An inverse Gaussian process model for degradation data." Technometrics 52.2 (2010): 188-197.

Wang, Chaonan, Liudong Xing, and Gregory Levitin. "Reliability analysis of multi-trigger binary systems subject to competing failures." Reliability Engineering \& System Safety 111 (2013): 917.

Wang, Fu-Kwun, and Tao-Peng Chu. "Lifetime predictions of LED-based light bars by accelerated degradation test." Microelectronics Reliability 52.7 (2012): 1332-1336.

Wang, Lizhi, et al. "A Bayesian reliability evaluation method with integrated accelerated degradation testing and field information." Reliability Engineering \& System Safety 112 (2013): 38-47.

Wang, Zhonglai, et al. "An approach to reliability assessment under degradation and shock process." Reliability, IEEE Transactions on 60.4 (2011): 852-863.

Whitmore, G. A. "Estimating degradation by a Wiener diffusion process subject to measurement error." Lifetime data analysis 1.3 (1995): 307-319.

Whitmore, G. A., and Fred Schenkelberg. "Modelling accelerated degradation data using Wiener diffusion with a time scale transformation." Lifetime data analysis 3.1 (1997): 27-45.

Wortman, M. A., Georgia-Ann Klutke, and Hayriye Ayhan. "A maintenance strategy for systems subjected to deterioration governed by random shocks." Reliability, IEEE Transactions on 43.3 (1994): 439-445.

$\mathrm{Wu}$, Shuen-De, et al. "Bearing fault diagnosis based on multiscale permutation entropy and support vector machine." Entropy 14.8 (2012): 1343-1356.

Xing, Liudong, and Joanne Bechta Dugan. "Analysis of generalized phased-mission system reliability, performance, and sensitivity." Reliability, IEEE Transactions on 51.2 (2002): 199-211.

Xing, Liudong, and Joanne Bechta Dugan. "A separable ternary decision diagram based analysis of generalized phased-mission reliability." Reliability, IEEE Transactions on 53.2 (2004): 174184.

Xing, Liudong, and Gregory Levitin. "Combinatorial analysis of systems with competing failures subject to failure isolation and propagation effects." Reliability Engineering \& System Safety 95.11 (2010): 1210-1215.

Xing, Liudong, and Gregory Levitin. "BDD-based reliability evaluation of phased-mission systems with internal/external common-cause failures." Reliability Engineering \& System Safety 112 (2013): 145-153.

Xing, Liudong, Chaonan Wang, and Gregory Levitin. "Competing failure analysis in non-repairable binary systems subject to functional dependence." Proceedings of the Institution of Mechanical Engineers, Part O: Journal of Risk and Reliability 226.4 (2012): 406-416.

Yang, Guangbin. "Environmental-stress-screening using degradation measurements." Reliability, IEEE Transactions on 51.3 (2002): 288-293.

Yang, Won Seok, Dae E. Lim, and Kyung C. Chae. "Maintenance of multi-state production systems deteriorated by random shocks and production." Journal of Systems Science and Systems Engineering 20.1 (2011): 110-118.

Yang, Qingyu, Nailong Zhang, and Yili Hong. "Reliability analysis of repairable systems with dependent component failures under partially perfect repair." Reliability, IEEE Transactions on 62.2 (2013): 490-498.

Yang, Kai, and Guangbin Yang. "Degradation reliability assessment using severe critical values." International Journal of Reliability, Quality and Safety Engineering 5.01 (1998): 85-95.

Ye, Zhi Sheng, Loon Ching Tang, and Hai Yan Xu. "A distribution-based systems reliability model under extreme shocks and natural degradation." Reliability, IEEE Transactions on 60.1 (2011): 246-256.

Ye, Zhi-Sheng, and Min Xie. "Stochastic modelling and analysis of degradation for highly reliable products." Applied Stochastic Models in Business and Industry 31.1 (2015): 16-32. 
Ye, Zhi-Sheng, and Nan Chen. "The inverse Gaussian process as a degradation model." Technometrics 56.3 (2014): 302-311.

Ye, Zhi-Sheng, et al. "Accelerated degradation test planning using the inverse Gaussian process." Reliability, IEEE Transactions on 63.3 (2014): 750-763.

$\mathrm{Ou}$, Yong, and Joanne Bechta Dugan. "Modular solution of dynamic multi-phase systems." Reliability, IEEE Transactions on 53.4 (2004): 499-508.

$\mathrm{Yu}, \mathrm{I}$. , and Che-Lun Chang. "Applying bayesian model averaging for quantile estimation in accelerated life tests." Reliability, IEEE Transactions on 61.1 (2012): 74-83.

Zang, Xinyu, Hairong Sun, and Kishor S. Trivedi. "A BDD-based algorithm for reliability analysis of phased-mission systems." Reliability, IEEE Transactions on 48.1 (1999): 50-60.

Zequeira, Rómulo I., and Christophe Bérenguer. "On the inspection policy of a two-component parallel system with failure interaction." Reliability Engineering \& System Safety 88.1 (2005): 99-107.

Zhang, Qian. "Case study of cost benefits of condition based maintenance used in medical devices." Reliability and Maintainability Symposium (RAMS), 2013 Proceedings-Annual. IEEE, 2013.

Zhao, Fuqiong, et al. "An Integrated Prognostics Method Under Time-Varying Operating Conditions." Reliability, IEEE Transactions on 64.2 (2015): 673-686.

Zhou, Y., et al. "Application of the horizontal slice of cyclic bispectrum in rolling element bearings diagnosis." Mechanical Systems and Signal Processing 26 (2012): 229-243.

Žliobaitè, Indrè, et al. "Active learning with evolving streaming data." Machine Learning and Knowledge Discovery in Databases. Springer Berlin Heidelberg, 2011. 597-612.

Zio, Enrico, and G. E. Apostolakis. "Two methods for the structured assessment of model uncertainty by experts in performance assessments of radioactive waste repositories." Reliability Engineering \& System Safety 54.2 (1996): 225-241.

Zio, Enrico. "Prognostics and health management of industrial equipment." Diagnostics and Prognostics of Engineering Systems: Methods and Techniques (2012): 333-356.

Zio, Enrico, and Michele Compare. "Evaluating maintenance policies by quantitative modeling and analysis." Reliability Engineering \& System Safety 109 (2013): 53-65. 